\title{
Analysis of complications related to dialysis catheters in cancer patients
}

\author{
Edwiges Ita de Miranda Moura ${ }^{1 *}$, Germana Alves de Brito ${ }^{1}$, Juliana Vendramini Cordeiro Fernandes², \\ Lilian Guardian², Joubert Araujo Alves ${ }^{1}$, Fabiana Plaza Sequeira², Marina Harume Imanishe ${ }^{1}$ and \\ Benedito Jorge Pereira'
}

\begin{abstract}
Background: Data on renal replacement therapy (RRT) in cancer patients with acute kidney injury (AKI) in the intensive care unit (ICU) and how these factors influence the efficiency of dialysis and the outcomes of these patients are scarce. We aim to describe the predictive clinical characteristics of hemodialysis catheter-related complications in critically ill cancer patients with acute kidney injury.
\end{abstract}

Methods: This is a retrospective cohort study of 62 cancer patients subjected to short-term hemodialysis catheter implants. We evaluated the clinical characteristics of patients and the complications related to catheter implantation: mild malfunction, characterized by reversal of access lines; infections; and catheter replacement due to severe malfunction or infections. The outcomes analyzed were recovery of renal function, discharge from the ICU, and death.

Results: The most frequent complications were related to mild malfunction, which justified dialysis line reversal in $21.3 \%$ of the patients. The complication rate was higher in patients undergoing conventional hemodialysis, in those with coronary disease, and in those who had more than three dialysis sessions. The presence of metastasis was more frequently related to catheter reversals. Patients with mild or severe malfunction were more likely to be hospitalized for more than 18 days in the ICU. All patients with mild malfunction had hospitalization times greater than 28 days.

Conclusions: Hemodialysis catheter-related complications were associated with longer ICU and hospital stays in cancer patients with AKI. Mild malfunction of the hemodialysis catheter was the most frequent complication. Patients with metastasis and sepsis, who used diuretics, and who used intermittent dialysis methods had milder catheter malfunctions.

Keywords: Renal insufficiency, Dialysis, Cancer care facilities, Catheters

\section{Background}

Acute kidney injury (AKI) is a common complication in cancer patients and may occur as a consequence of the underlying disease or treatment-related complications, such as tumor lysis syndrome [1] and drug-induced nephropathy [2], and may be secondary to surgical procedures [3]. In addition, one of the main etiologies of AKI in cancer patients is sepsis, which induces renal

\footnotetext{
* Correspondence: edwiges_ita@hotmail.com

${ }^{1}$ Department of Nephrology, A. C. Camargo Cancer Center, São Paulo, SP 01509-010, Brazil

Full list of author information is available at the end of the article
}

hypoperfusion secondary to systemic vasodilation and renal vasoconstriction [4].

Data on renal replacement therapy (RRT) in cancer patients with acute kidney injury (AKI) in the intensive care unit (ICU) are scarce, and intermittent hemodialysis complications such as hypotension and clotting are common in some studies. For example, in one study, hypotension occurred in $25 \%$ of the sessions, clotting in $23.8 \%$, and malfunctioning catheters in $29.2 \%$ of the procedures [5]. The clinical manifestations of these dysfunctions are usually absent or reduced blood flow, negative arterial pressure in the extracorporeal system, and collapse of the arterial segment of the extracorporeal 
system. The reversal of dialysis blood lines is a procedure used when the blood flow during hemodialysis is low or when venous or arterial bloodline pressures are high. Although it is not a direct complication, dialysis blood line reversal represents a mild malfunction of the catheter that does not interrupt the procedure. Severe malfunction is characterized by the absence of blood flow in the extracorporeal system and the mild malfunction with a reversal of lines. Most catheters inserted into central veins develop fibrin clots one to several weeks after implantation and are silent at first, until they obstruct the distal part of the catheter; this clot development can be minimized by the conventional use of a heparin lock to prevent access thrombosis between hemodialysis sessions [6].

The objective of this study was to describe the clinical characteristics of hemodialysis catheter-related complications due to mild or severe malfunction of the access and infection in critically ill cancer patients with AKI and to evaluate the influence of clinical characteristics associated with the underlying oncological disease and other chronic comorbidities on the progression of these dialysis patients with complications related to the use of the dialysis catheter.

\section{Methods}

This was a retrospective cohort study based on a review of medical records, which evaluated the 62 patients who underwent vascular access implantation for hemodialysis at the AC Camargo Cancer Center from January 2011 to December 2013. The study included patients older than 18 years, with cancer that had been treated or was under treatment at the hospital, who presented AKI as defined in the KDIGO Guidelines for AKI [7] as stage 3, i.e., having an increase in serum creatinine greater than $300 \%(>3 \times)$ relative to baseline or greater than or equal to $4 \mathrm{mg} / \mathrm{dL}$, with an acute increase of at least $0.5 \mathrm{mg} / \mathrm{dL}$, and a urinary flow of less than $0.3 \mathrm{ml} / \mathrm{kg} / \mathrm{h}$ for $24 \mathrm{~h}$ or anuria in $12 \mathrm{~h}$. All patients had indications for dialysis by the nephrology team who cared for them and underwent temporary catheter implantation for hemodialysis at admission. The catheters were all $20 \mathrm{~cm} / 12 \mathrm{Fr}$ (Arrow International, Inc.). Patients who did not have cancer, those who had long-term hemodialysis catheters, and those who did not undergo access implantation within the hospital were excluded from the study.

The electronic file system of the AC Camargo Cancer Center was used to locate the sample by observing the item "vascular access implantation for hemodialysis" in all patients with dialytic renal dysfunction admitted to the ICU within a 2-year period, followed by subsequent searches of the clinical and laboratory data of these patients. Data were collected after submission of the study protocol to the hospital's Ethics and Research Commission. The study was registered under number CAAE 32913714.3.0000.5432.

The demographic characteristics of the patients, such as age and gender; the clinical characteristics of the underlying neoplasia (type of neoplasia, whether solid or hematologic, and the presence of metastases); the type of treatment instituted (surgery, chemotherapy, radiotherapy); the performance status evaluated by the Zubrod scale (Eastern Cooperative Oncology Group, ECOG) [8]; previous comorbidities, such as systemic arterial hypertension $(\mathrm{SAH})$, diabetes mellitus, congestive heart failure, and coronary disease; the AKI etiology (sepsis, nephrotoxicity, obstructive, multifactorial); the factors related to the access implantation for dialysis, such as the site (jugular, femoral) and insertion side; and the type of dialysis, such as conventional hemodialysis, extended or sustained low-efficiency dialysis (SLED), and continuous venovenous hemodiafiltration, were evaluated. The complications evaluated in this study related to catheter implantation were mild malfunction of catheter, characterized by reversal of access lines during dialysis that did not interrupt the procedure; infections; and catheter replacement due to severe malfunction of the catheter or infections. The outcomes analyzed were recovery of renal function, discharge from the ICU, and death.

The Statistical Package for the Social Sciences (SPSS) version 15.0 for Windows (SPSS Inc., Chicago, IL, USA) was used. Qualitative variables are presented as absolute (n) and relative (\%) frequencies and quantitative variables as means and standard deviations. The presence of associations between discharge or death and other categorical variables was assessed using the chi-squared test and, when necessary, the chi-squared test of the likelihood ratio. The level of significance adopted was 0.05 $(\alpha=5 \%)$; descriptive levels (p) below this value were considered significant.

\section{Results \\ Patient characteristics}

The electronic records of 62 cancer patients hospitalized in the ICU with a diagnosis of AKI undergoing dialysis treatment were analyzed. The mean age of the patients was $63.9 \pm 13.7$ years, and $59.7 \%$ were male and $40.3 \%$ female. Solid tumors were the most frequent type of neoplasia $(83.9 \%)$, and only $16.1 \%$ of the patients had hematologic diseases. In addition, $53.2 \%$ of the patients had at least one metastasis. Most of the patients (82\%) had other morbidities, with SAH (41.9\%) being the most prevalent. When classified according to ECOG, 7 (11.3\%) patients belonged to ECOG 0, 28 (45.2\%) to ECOG 1, 13 (20.9\%) to ECOG 2, 12 (19.4\%) to ECOG 3, $2(3.2 \%)$ to ECOG 4, and none to ECOG 5. Regarding the oncological treatment modalities instituted, surgery 
(74.1\%) was the most used therapeutic resource. These data are listed in Table 1.

It was observed that $61.3 \%$ of the patients required mechanical ventilation $(n=38)$ and that sepsis occurred in $32.2 \%$ of the patients $(n=20)$. Among the dialysis therapies used for the treatment of AKI, the use of intermittent methods was more frequent, with conventional hemodialysis $(n=41,67.2 \%)$ being the most frequently performed modality (Table 1 ).

The mean length of ICU stay was $15.5 \pm 11.5$ days, and the mean length of hospital stay was $44.0 \pm 34.6$ days. The mean permanence time of the hemodialysis catheter in the study patients was 18 days. The most frequent complications were related to mild malfunction, which justified dialysis line reversal in $21.3 \%$ of the patients. These data are listed in Table 2.

\section{Influence of clinical characteristics and complications on the vascular access}

Analysis of the access complications showed that mild malfunction was the most frequent, occurring in 13 patients $(21.3 \%)$. The presence of metastasis was more frequently associated with catheter reversal $(31.3 \%$ in

Table 1 Demographic and clinical characteristics of cancer patients with AKI admitted to the ICU

\begin{tabular}{|c|c|c|}
\hline Characteristics & $n$ & $\%$ \\
\hline Male & 37 & 59.7 \\
\hline Female & 25 & 40.3 \\
\hline \multicolumn{3}{|l|}{ Performance status } \\
\hline ECOG 3 & 12 & 19.3 \\
\hline \multicolumn{3}{|l|}{ Baseline neoplasia } \\
\hline Solid tumor & 52 & 83.9 \\
\hline Hematologic disease & 10 & 16.1 \\
\hline Presence of metastasis & 33 & 53.2 \\
\hline \multicolumn{3}{|l|}{ Cause of AKI } \\
\hline Sepsis AKI & 45 & 72.5 \\
\hline Sepsis + Nephrotoxicity AKI & 4 & 6.4 \\
\hline Nephrotoxicity AKI & 1 & 1.6 \\
\hline Use of diuretics & 5 & 8.1 \\
\hline \multicolumn{3}{|l|}{ Dialysis } \\
\hline Conventional HD & 41 & 66.1 \\
\hline SLED & 37 & 60.7 \\
\hline Continuous HD & 12 & 19.7 \\
\hline \multicolumn{3}{|l|}{ Clinical progression } \\
\hline ICU discharge & 33 & 53.2 \\
\hline ICU death & 29 & 46.8 \\
\hline Renal function recovery & 24 & 42.8 \\
\hline Palliative care & 20 & 32.2 \\
\hline
\end{tabular}

$H D$ hemodialysis, ECOG Eastern Cooperative Oncology Group, ICU intensive care unit, SLED sustained low-efficiency dialysis
Table 2 Sites of dialysis catheter insertion and frequencies of access-related complications in cancer patients with AKI

\begin{tabular}{lll}
\hline Variable & $\mathrm{n}$ & $\%$ \\
\hline Insertion site & 26 & \\
Jugular & 14 & 22.6 \\
Right jugular & 12 & 19.6 \\
Left jugular & 26 & 42.6 \\
Femoral & 15 & 24.5 \\
Right femoral & 11 & 18.0 \\
Left femoral & & \\
Events/Complications & 13 & 21.3 \\
Dialysis line reversal & 10 & 16.4 \\
HD catheter replacement & 18 & 29.5 \\
No. of sessions with same catheter & 10 & 16.4 \\
$\leq 2$ & 23 & 44.3 \\
Between 3 and 7 & 8 & 13.1 \\
$\geq 8$ & &
\end{tabular}

patients with metastasis vs $10.3 \%$ in patients with no metastasis, $p<0.05$ ), and these reversals were proportional to the number of metastases. In addition, the complication rate was higher in patients undergoing conventional hemodialysis $(29.3 \%$ vs $5 \%)$. The risk of mild malfunction was higher in patients who had coronary artery disease (57.1\% vs $16.7 \%)$ and who underwent more than three dialysis sessions. Septic patients had more dialysis mild malfunction (5\%). It should be emphasized that the site of access implantation had no influence on line reversals. In addition, the use of diuretics after onset of AKI was a factor that contributed to an increase in dialysis catheter line reversals.

The catheter was replaced in 10 patients $(16.7 \%)$, all of whom were older than 60 years, had ECOG status 3, were on mechanical ventilation for more than 2 days, and had more than three metastases. Those patients who needed catheter replacement due to severe malfunction included two implanted in the right femoral vein, one with hematologic disease, and one with a metastatic solid tumor. Only one patient needed catheter replacement due to infection. This patient had hematologic disease and associated coronary disease and was implanted in the right femoral vein. Due to the rarity of infection-related complications (one event) and catheter malfunction (two events), it was not possible to analyze their predictors. The other cases of catheter replacement occurred due to replacement for a tunneled catheter in five patients and due to accidental exteriorization in two. None of the 19 patients who progressed to palliative care needed the dialysis catheter replaced (Table 3 ).

We next compared the need for catheter line reversal and replacement between the groups of patients with $\leq 3$ 
Table 3 Need for catheter line reversal due mild malfunction of catheter during hemodialysis in cancer patients with AKI in the ICU

\begin{tabular}{|c|c|c|c|c|c|}
\hline \multirow[t]{2}{*}{ Clinical parameters } & \multicolumn{2}{|c|}{ No reversal } & \multicolumn{2}{|c|}{ With reversal } & \multirow[b]{2}{*}{$\mathrm{p}$} \\
\hline & $\mathrm{n}$ & $\%$ & $\mathrm{n}$ & $\%$ & \\
\hline Metastasis & 3 & 9.09 & 10 & 31.3 & $0.046^{*}$ \\
\hline \multicolumn{6}{|l|}{ Comorbidities } \\
\hline $\mathrm{SAH}$ & 22 & 84.6 & 4 & 15.4 & 0.330 \\
\hline $\mathrm{DM}$ & 12 & 92.3 & 1 & 7.70 & 0.176 \\
\hline $\mathrm{CHF}$ & 6 & 60.0 & 4 & 44.4 & 0.066 \\
\hline CKD & 12 & 85.7 & 2 & 14.3 & 0.465 \\
\hline AMI/Coronary insufficiency & 3 & 42.8 & 4 & 57.1 & $0.014^{*}$ \\
\hline Stroke & 3 & 100 & 0 & 0.00 & 0.454 \\
\hline \multicolumn{6}{|l|}{ Treatment performed } \\
\hline Surgery & 32 & 74.4 & 11 & 26.2 & 0.308 \\
\hline Radiotherapy & 7 & 70.0 & 3 & 30.0 & 0.521 \\
\hline Chemotherapy & 14 & 73.6 & 5 & 27.8 & 0.425 \\
\hline Use of diuretics & 2 & 40.0 & 3 & 75.0 & $0.007^{*}$ \\
\hline \multicolumn{6}{|l|}{ Clinical complications } \\
\hline Sepsis & 19 & 95.0 & 1 & 5 & $0.030^{*}$ \\
\hline Respiratory insufficiency & 13 & 86.6 & 2 & 14.3 & 0.465 \\
\hline Delirium & 1 & 50.0 & 1 & 50.0 & 0.314 \\
\hline MV & 30 & 78.9 & 8 & 21.1 & 0.949 \\
\hline \multicolumn{6}{|l|}{ Dialysis mode } \\
\hline Conventional HD & 29 & 70.7 & 12 & 29.3 & $0.030^{*}$ \\
\hline SLED & 27 & 72.9 & 10 & 27.0 & 0.176 \\
\hline Continuous HD & 9 & 75.0 & 3 & 25.0 & 0.728 \\
\hline \multicolumn{6}{|l|}{ Clinical progression } \\
\hline ICU death & 25 & 86.2 & 4 & 13.8 & 0.172 \\
\hline Renal function recovery & 19 & 79.1 & 5 & 20.8 & 0.925 \\
\hline ICU discharge & 23 & 71.8 & 9 & 28.1 & 0.158 \\
\hline Palliative care & 15 & 75.0 & 5 & 26.3 & 0.521 \\
\hline
\end{tabular}

$A K I$ acute kidney injury, $A M I$ acute myocardial infarction, $C H F$ congestive heart failure, CKD chronic kidney disease, DM diabetes mellitus, ECOG Eastern Cooperative Oncology Group, HD hemodialysis, SAH systemic arterial hypertension, SLED sustained low-efficiency dialysis, $M V$ mechanical ventilation. ${ }^{*} p<0.05$

vs $>3$ dialysis sessions with the same catheter, use of vascular access $\leq 15$ days vs $>15$ days, length of ICU stay $\leq 18$ days vs $>18$ days, and length of hospital stay $\leq 28$ days vs $>28$ days. Patients who underwent more than three dialysis sessions with the same catheter experienced more vascular access line reversals than patients who underwent three or fewer ( $48 \%$ vs $2.8 \%, p=0.005$ ), but these two groups had no significant difference in the need for catheter replacement ( 24.0 vs $11.1 \%, p=0.181$ ). Patients who had the same catheter for more than 15 days experienced fewer mild malfunctions than patients who had the same catheter for 15 days or less (23.5\% vs $76.5 \%, p=0.003)$, but these two groups had no significant difference in the rate of catheter replacement (5.9\% vs $21.2 \% p=0.161$ ). Patients who stayed more than 18 days in the ICU were more likely to need catheter line reversal than patients who stayed $\leq 18$ days ( 44.4 vs $11.9 \%, p=0.005)$. These two groups also showed a significant difference in the need catheter replacement (33.3 vs $9.5 \%, p=0.023$ ). Taking into account a length of hospital stay greater than 28 days, all 13 patients with mild malfunction had hospital stays greater than 28 days $(32.5 \%$ vs $0 \%, p=0.005)$. The data for these categorizations and mild malfunction are summarized in Table 4.

\section{Discussion}

AKI is associated with higher morbidity and mortality in critically ill cancer patients [9]. Thus, the identification and prevention of complications related to the hemodialysis catheter are fundamental for improving patient care. This study evaluated the clinical predictors of hemodialysis catheter-related complications in critically ill cancer patients.

Most of the patients had solid tumors, for whom surgery was the most common treatment, and $82 \%$ had other comorbidities, with SAH being the most common (41.9\%). The most commonly used dialysis treatment was the intermittent method, and sepsis was the most frequent AKI etiology. These data corroborate those reported in other studies, which also showed a 78\% prevalence of sepsis in cancer patients with AKI admitted to the ICU. Similarly, other studies demonstrated that sepsis was the main cause of AKI, occurring in $51 \%$ of the 149 critically ill cancer patients evaluated in a prospective cohort $[10,11]$.

The presence of metastasis, the use of intermittent dialysis methods, the presence of sepsis, and the use of diuretics after the onset of AKI were more frequent in patients with mild malfunction of hemodialysis catheter. In fact, the neoplasia progression stage and the sites of metastases seem to contribute to hypercoagulability and a higher risk of thrombosis [12, 13]. Sepsis also constitutes a state of hypercoagulability, characterized by several factors: the generation of thrombin over a few hours in response to the presence of endotoxin and tumor

Table 4 Need for catheter line reversal because of mild malfunction of the catheter, and the lengths of ICU and hospital stays

\begin{tabular}{|c|c|c|c|c|c|c|c|}
\hline \multirow[t]{2}{*}{ Parameters } & \multicolumn{2}{|c|}{ Overall } & \multicolumn{2}{|c|}{ No reversal } & \multicolumn{2}{|c|}{ With reversal } & \multirow[b]{2}{*}{$p$} \\
\hline & $\mathrm{n}$ & $\%$ & $n$ & $\%$ & $\mathrm{n}$ & $\%$ & \\
\hline Catheter use $>15$ days & 17 & 27.4 & 13 & 76.5 & 4 & 23.5 & $0.003^{*}$ \\
\hline More than 3 ト & 25 & 40.3 & 13 & 52.0 & 12 & 48.0 & $<0.005^{*}$ \\
\hline CU sta & 18 & 29.0 & 10 & 55.6 & 8 & 44.4 & $0.005^{*}$ \\
\hline Hospital stay $>28$ days & 40 & 64.5 & 27 & 67.5 & 13 & 32.5 & $0.005^{*}$ \\
\hline
\end{tabular}


necrosis factor; a reduction in the action of endogenous anticoagulants, such as tissue factor pathway inhibitor, activated $\mathrm{C}$ protein and antithrombin; resistance to fibrinolysis; and endothelial damage. Thus, it is postulated that this factor may also contribute to catheter malfunction and the higher rate of line reversal [14]. It should be noted that among the complications studied, only access line reversal is considered a nursing intervention, which is performed autonomously and occurs due to the lack of flow in the vascular access. Thus, this practice favors blood flow in the access, but it reduces the efficiency of dialysis by allowing recirculation of the dialyzed blood [15].

The access implant site had no influence on either line reversals or catheter replacement; however, a trend toward greater malfunction frequency was observed when the catheter was implanted in the right femoral vein. This finding has also been reported in other studies [7, 16] that note that the right femoral access, in addition to being associated with a higher incidence of infection, may be associated with a higher incidence of dysfunction. In part, it is for this reason that the KDIGO AKI guideline [7] recommends the right internal jugular vein as the first-choice access, followed by the femoral vein, left jugular vein and the subclavian veins. It should be noted that in our patients, there was a low rate of infection related to vascular access, which was observed in only one patient. This finding indicates the effectiveness of interventions to reduce the rate of infections in the ICU, which include proper hand hygiene, maximum barrier precaution at the time of catheter insertion (use of a sterile apron, mask, glove, and cap), use of sterile devices, and skin antisepsis with chlorhexidine in the indicated area, among other measures [16].

Another complication that may hamper renal function recovery and prolong the treatment or hospitalization time is the occurrence of severe hemodialysis malfunction. The overall rate of catheter replacement was $16.4 \%$, with $3.3 \%$ due to malfunction and $1.6 \%$ due to infection. According to a previous study, the conditions associated with this complication include fibrin sheath formation, thrombus within the catheter, and catheter kinks (catheter fracture or disconnection, catheter malposition or migration, and catheter tip adherent to the vessel wall) [17]. In the present study, age greater than 60 years, ECOG status of 3, and use of MV for more than $48 \mathrm{~h}$ were predictors of catheter replacement. In fact, these variables characterized a greater severity of the clinical picture, thus contributing to a higher frequency of catheter replacement.

It should be noted that the hemodialysis catheterrelated complications were associated with longer stays in the ICU and hospital. In fact, according to an analysis performed in a previous study, the management of vascular access failure is associated with not only the length of hospital stay but also hospital expenses [18]. Thus, it is important to establish measures to prevent catheter dysfunction, such as anticoagulation care in the dialysis system, adequate selection of the catheter material, and use of antisepsis techniques during insertion [19].

This study had some limitations. First, the data were obtained by searching medical records; no clinicalpicture severity scores were evaluated. In addition, considering the low frequencies of some catheter-related complications, the study did not have enough statistical power to discriminate all of the predictors evaluated. Additionally, the effect of catheter line reversal on dialysis efficiency via measurement of the effective dialysis dose was not evaluated.

\section{Conclusion}

Mild malfunction of the hemodialysis catheter was the complication most frequently associated with hemodialysis access. The access implantation site had no influence on line reversal. The patients with metastasis or sepsis, use of diuretics, or use of intermittent dialysis methods had more frequent mild catheter malfunction. The need for catheter replacement was observed in patients who were older than 60 years, with ECOG status of 3 , use of mechanical ventilation for more than 2 days, and more than three metastases. In addition, patients with mild and severe malfunction of the dialysis catheter had longer ICU and hospital stays. The occurrence of only one infectious complication reflected the efficacy of the precautionary measures adopted in the ICU studied, even among immunosuppressed cancer patients.

\section{Abbreviations \\ AKI: Acute kidney injury; AMI: Acute myocardial infarction; CD: Coronary disease; CHF: Congestive heart failure; CKD: Chronic kidney disease; \\ DM: Diabetes mellitus; ECOG: Eastern Cooperative Oncology Group; HD: Hemodialysis; ICU: Intensive care unit; KDIGO: Kidney Disease: Improving Global Outcomes; MV: Mechanical ventilation.; RDBL: Reversal of dialysis \\ blood lines; RRT: Renal replacement therapy; SAH: Systemic arterial hypertension; SLED: Extended or sustained low-efficiency dialysis \\ Acknowledgments \\ Not applicable. \\ Funding \\ We did not receive any specific grant from funding agencies. \\ Availability of data and materials \\ Please contact author for data requests. \\ Authors' contributions \\ EIMM, GAB, JVC, LG, JAA, FPS and BJP participated in the study design and development; collecting, processing, analyzing, and interpreting data; \\ bibliographical research; and manuscript preparation and revision. All authors} read and approved the final manuscript.

Authors' information

Not applicable. 


\section{Ethics approval and consent to participate}

Data were collected after submission of the study protocol to the A.C. Camargo Cancer Center Ethics and Research Commission. The study was registered under number CAAE 32913714.3.0000.5432.

\section{Consent for publication}

Not applicable

\section{Competing interests}

The authors declare that they have no competing interests.

\section{Publisher's Note}

Springer Nature remains neutral with regard to jurisdictional claims in published maps and institutional affiliations.

\section{Author details}

${ }^{1}$ Department of Nephrology, A. C. Camargo Cancer Center, São Paulo, SP 01509-010, Brazil. Department of Critical Care, A. C. Camargo Cancer Center, São Paulo, SP 01509-010, Brazil.

Received: 28 September 2017 Accepted: 12 January 2018

\section{Published online: 22 January 2018}

\section{References}

1. Wilson FP, Berns JS. Onco-nephrology: tumor lysis syndrome. Clin J Am Soc Nephrol. 2012:7:1730-9.

2. Maccariello E. Contrast induced nephropathy. J Bras Nefrol. 2016;38:388-9.

3. De Tommasi C, Cusimano MD. Rhabdomyolysis after neurosurgery: a review and a framework for prevention. Neurosurg Rev. 2013;36:195-202.

4. Valette X, Du Cheyron D. A critical appraisal of the accuracy of the RIFLE and AKIN classifications in defining "acute kidney insufficiency" in critically ill patients. J Crit Care. 2013;28:116-25.

5. Da Costa e Silva VT, Costalonga EC, Oliveira APL, Hung J, Caires RA, Hajjar LA, Fukushima JT,2 Soares CM, Bezerra JS, Oikawa L, Yu L, Burdmann EA. Evaluation of intermittent Hemodialysis in critically ill cancer patients with acute kidney injury using single-pass batch equipment. PLoS One. 2016; 11(3): e0149706.

6. Liu J, Wang C, Zhao H, Zhang J, Ma J, Hou Y, et al. Anticoagulant therapies versus heparin for the prevention of hemodialysis catheter-related complications: systematic review and meta-analysis of prospective randomized controlled trials. Int J Clin Exp Med. 2015;8:11985-95.

7. Kidney Disease: Improving Global Outcomes (KDIGO) Acute Kidney Injury Work Group. KDIGO Clinical Practice Guideline for Acute Kidney Injury. Kidney Inter., Suppl. 2012; 2: 1-138.

8. Oken MM, Creech RH, Tormey DC, Horton J, Davis TE, McFadden ET, et al. Toxicity and response criteria of the eastern cooperative oncology group. Am J Clin Oncol. 1982;5:649-55.

9. Córdova-Sánchez BM, Herrera-Gómez Á, Namendys-Silva SA. Acute kidney injury classified by serum creatinine and urine output in critically ill cancer patients. Biomed Res Int. 2016;2016:7.

10. Maccariello E, Valente C, Nogueira L, Bonomo H, Ismael M, Machado JE, et al. Outcomes of cancer and non-cancer patients with acute kidney injury and need of renal replacement therapy admitted to general intensive care units. Nephrol Dial Transplant. 2011;26:537-43.

11. Da Costa ESVT, Costalonga EC, Oliveira AP, Hung J, Caires RA, Hajjar LA, et al. Evaluation of intermittent hemodialysis in critically ill cancer patients with acute kidney injury using single-pass batch equipment. PLoS One. 2016;11: E0149706.

12. Zhu YW, Feng TB, Zhou XJ, Hu XL, Ding J, Zhu WY, et al. Routine hemostasis and hemogram parameters: valuable assessments for coagulation disorder and chemotherapy in cancer patients. Chin Med J. 2016;129:1772-7.

13. Kirwan CC, Clarke AC, Howell SJ, Castle J. PO-31 - circulating tumour cells and hypercoagulability: a lethal relationship in metastatic breast cancer. Thromb Res. 2016;140:S188.

14. Semeraro N, Ammollo CT, Semeraro F, Colucci M. Coagulopathy of acute sepsis. Semin Thromb Hemost. 2015;41:650-8.

15. Bander JB, Schwab SJ, Woo K. Central catheters for acute and chronic hemodialysis access. 2016. https://www.uptodate.com/contents/ central-catheters-for-acute-and-chronic-hemodialysis-access?search =Central\%20catheters\%20for\%20acute\%20and\%20chronic\%20
hemodialysis\%20access.\&source=search_result\&selectedTitle=1 150\&usage_type=default\&display_rank=. Accessed 23 Dec 2016.

16. Clark EG, Barsuk JH. Temporary hemodialysis catheters: recent advances. Kidney Int. 2014;86:888-95.

17. Bream PR. Update on insertion and complications of central venous catheters for hemodialysis. Semin Intervent Radiol. 2016;33:31-8.

18. Sawant A, Mills PK, Dhingra H. Increased length of stay and costs associated with inpatient management of vascular access failures. Semin Dial. 2013;26:106-10.

19. O'Grady NP, Alexander M, Burns LA, Dellinger EP, Garland J, Heard SO, et al. Guidelines for the prevention of intravascular catheter-related infections. Am J Infect Control. 2011;39:S1-34.

\section{Submit your next manuscript to BioMed Central and we will help you at every step:}

- We accept pre-submission inquiries

- Our selector tool helps you to find the most relevant journal

- We provide round the clock customer support

- Convenient online submission

- Thorough peer review

- Inclusion in PubMed and all major indexing services

- Maximum visibility for your research

Submit your manuscript at www.biomedcentral.com/submit
) Biomed Central 